\title{
RECURSOS FISIOTERAPÊUTICOS EM TUBERCULOSE PULMONAR
}

\author{
Physical therapy for pulmonary tuberculosis
}

\author{
Daniela Carla de Oliveiral, Cirana de Moura Coutinho², Gisele Leiko Takata2, \\ Rachel de Castro Schiaveto ${ }^{2}$, Maria Nazareth Vianna Roseiro ${ }^{3}$
}

\begin{abstract}
RESUMO
A Tuberculose Pulmonar, segundo a Organização Mundial de Saúde, mata 2 milhões de pessoas a cada ano, em todo o mundo. Objetivo: Verificar na literatura indexada os recursos fisioterapêuticos empregados no tratamento da Tuberculose Pulmonar. Metodologia: Levantamento bibliográfico através da PUBMED, utilizando a combinação das palavras "physical therapy and pulmonary tuberculosis", obedecendo aos critérios de inclusão: publicações no período de 1997 a 2004 em inglês, com pesquisas realizadas em seres humanos, sem distinção de idade ou sexo, a partir do resumo (abstract). Resultados: Encontrados 26 artigos, porém, somente 9 pesquisas abordaram recursos fisioterapêuticos. O ultra-som é o recurso mais utilizado, porém há indicação de exercícios de relaxamento e de condicionamento, treino da musculatura respiratória e uso de Laser As-Ga. A necessidade de aprofundar os conhecimentos nessa matéria é urgente, considerando a importância da TP e o papel significativo da fisioterapia na melhora da qualidade de vida destes pacientes.

Palavras-chave: Tuberculose Pulmonar, Fisioterapia, Reabilitação Pulmonar, Ultra-Som, Laser As-Ga.

\section{SUMMARY}

According to the World Health Organization (WHO), two million people die of pulmonary tuberculosis worldwide every year. Objective: To verify the description of the physical therapy modalities employed in the treatment of pulmonary tuberculosis in the indexed literature. Methodology: Bibliographical survey through PUBMED, using the words combination "physical therapy and pulmonary tuberculosis". The following criteria of inclusion were applied for the search based on the abstract: (a) publications from 1997 to 2004, (b) in English, (c) studies carried out with human beings, (d) without distinction of age or sex. Results: 26 articles were found; only 9 of them addressed physical therapy intervention. Ultrasound is the most employed modality, but there are indications of relaxing and conditioning exercises, respiratory muscle training and the use of As-Ga Laser. The necessity of further knowledge on this subject is urgent, considering the importance of pulmonary tuberculosis among the infectious diseases and the significant role of physical therapy in improving the patients' quality of life.
\end{abstract}

Keywords: Pulmonary Tuberculosis, Physical therapy, Pulmonary Rehabilitation, Ultrasound, As-Ga Laser

\section{INTRODUÇÃO}

A Tuberculose Pulmonar (TP), segundo a Organização Mundial de Saúde (OMS), mata 2 milhões de pessoas a cada ano, em todo o mundo, apesar de a doença ser prevenida e curada, e desde 1993, é considerada como uma emergência global ${ }^{1}$.

A OMS estima que um teç̧o da população mundial está infectada pelo Mycobacterium tuberculosis, bactéria também denominada "bacilo de Koch"(BK). O Brasil ocupa o $13^{\circ}$ lugar em incidência desta doença com o índice de 129.000 casos por ano e, entre eles, apenas 90.000 são notificados ${ }^{1}$. Por ser um aeróbio estrito, o BK infecta preferencialmente os pulmões. A presença de oxigênio facilita sua multiplicação, e a ligação do órgão ao meio externo favorece sua transmissão ${ }^{2,3}$.

\footnotetext{
Trabalho realizado na Universidade de Ribeirão Preto - UNAERP, na $8^{\mathrm{a}}$ etapa do Curso de Fisioterapia, sem financiamento, e apresentado no XVI Congresso Brasileiro de Fisioterapia-COBRAF, em São Paulo-SP, 05 a 08 de outubro de 2005.

${ }^{1}$ Fisioterapeuta graduada pela Universidade de Ribeirão Preto _ UNAERP e acadêmica do Curso de Especialização em Fisioterapia de Hospital de Emergência UEHCFMRP-USP

${ }^{2}$ Fisioterapeuta graduada pela Universidade de Ribeirão Preto-UNAERP

${ }^{3}$ Fisioterapeuta. Mestre em Enfermagem em Saúde Pública pela EERP-USP. Professora Adjunta do Curso de Fisioterapia da Universidade de Ribeirão Preto-UNAERP
} 
O Mycobacterium tuberculosis se multiplica a cada 14 a 20 horas, o que explica a evolução lenta da doença e, também, a administração de medicamentos ser feita uma só vez ao dia ${ }^{3}$.

A propagação da TP se dá através do indivíduo bacilífero que, ao tossir, lança na atmosfera gotículas de secreção com bacilos (gotículas de Pflügge), que ao serem inalados, podem alcançar bronquíolos e alvéolos, iniciando assim a multiplicação bacilar. Em um ano, um indivíduo bacilífero pode infectar 10 a 15 pessoas $^{3,4,5}$.

O quadro clínico da TP é de evolução lenta. Os sintomas são tosse inicialmente seca e depois produtiva, hemoptise, febre baixa e vespertina, sudorese noturna, astenia, anorexia e perda ponderal $^{2,3,4}$.

O tratamento clínico da TP é baseado na administração de antibióticos resistentes ao bacilo ${ }^{2,3,4,5}$.

\section{OBJETIVO}

O objetivo do estudo é verificar na literatura indexada os recursos fisioterapêuticos empregados no tratamento da Tuberculose Pulmonar.

\section{METODOLOGIA}

A pesquisa consistiu em levantamento bibliográfico através da PUBMED, utilizando a combinação das palavras "physical therapy and pulmonary tuberculosis", em 25 de julho de 2005.

Os critérios de inclusão foram: publicações no período de 1997 a 2004 em inglês, com pesquisas realizadas em seres humanos, sem distinção de idade ou sexo, a partir do resumo (abstract).

\section{RESULTADOS}

Foram encontrados 26 artigos, porém, somente 9 pesquisas abordaram recursos fisioterapêuticos.

Lomachenkov et al. ${ }^{6}$ pesquisaram os efeitos do tratamento da TB utilizando drogas e microondas, e demonstraram a ação inibitória no bacilo após um mês de terapia.

Samosiuk et al. ${ }^{7}$ utilizaram o ultra-som de baixa frequiência (44 kHz, amplitude 2 microm) tanto na região da TB quanto em paravertebrais, além da terapia específica. Dados validam o uso do ultra-som como um coadjuvante no tratamento de novos casos de TB.

Para Balasaniants ${ }^{8}$, é indicada a utilização de radiação ultrasônica no baço em pacientes com tuberculose aguda progressiva, pois há uma melhora clínica e radiológica pulmonar, além da redução dos índices bioquímicos de inflamação.

Conforme relato de Savchenko \& Samosiuk ${ }^{9}$, pacientes com TB que receberam a terapia por ultra-som, além da quimioterapia, apresentaram sinais de melhora significativos tais como o desaparecimento mais rápido de importantes sintomas clínicos, marcas radiológicas de TB como alteração focal e infiltrativa e fechamento da cavidade, cessando a excreção da bactéria.

Porém, Klymenko ${ }^{10}$ demonstrou que podem ocorrer reações focais e sistêmicas de caráter provisório em decorrência do uso do ultra-som de baixa freqüência, mas não significam que sejam uma contra-indicação. As reações focais devem ser acompanhadas com exames radiográficos criteriosos, evitando dessa forma o agravamento do processo.

Tada et al. ${ }^{11}$ pesquisaram os efeitos de um programa de reabilitação pulmonar, por um período médio de 3,9 semanas, em pacientes internados com seqüela de TB pulmonar. O tratamento consistiu em relaxamento, exercícios de condicionamento, treinamento e reeducação da musculatura respiratória. Foi demonstrado aumento do VC $(1,481$ a 1,591), do VEF1 (0,931 a 1,021), da PaO2 (67,1Torr a 72,4 Torr), do teste de caminhada de 6 minutos (303m a 339m), da Pimax (38,5cmH2O a 47,5cmH2O), da tolerância ao exercício (19,6 pontos a 22,5 pontos), melhora dos sintomas de dispnéia $(18,4$ pontos a 22,5 pontos) e da qualidade de vida $(39,0$ pontos a 44,2 pontos). Os dados sugerem que a reabilitação pulmonar é indicada para melhorar a função pulmonar, a tolerância ao exercício, a diminuição de sintomas e a melhora na qualidade de vida de pacientes com seqüelas pulmonares decorrentes de TB.

Levechenko ${ }^{12}$ também realizou uma pesquisa com exercícios fisioterapêuticos sem especificá-los.

Puri \& Arora ${ }^{13}$ utilizaram Laser As-Ga (890nm) como coadjuvante da terapia medicamentosa, diminuindo os bacilos da TB e prevenindo o agravamento de mutantes resistentes com a melhora da secreção.

Um procedimento desenvolvido por Strelis et al. ${ }^{14}$ tem sido feito para reabilitação de pacientes com TB, após cirurgia de ressecção pulmonar, com a finalidade de prevenir complicações cirúrgicas tardias. Foram realizados 13 a 14 dias de fisioterapia sistêmica e localizada, associadas ao uso de agentes tuberculostáticos. A fisioterapia sistêmica consistiu na utilização de um dispositivo elétrico especial que envolve o tórax fazendo uma vibrocompressão. $\mathrm{Na}$ fisioterapia localizada, foi aplicada massagem local da raiz do pulmão operado, com um vibrador elétrico. Os autores afirmam que o procedimento estudado reduz a probabilidade de desenvolvimento de eventos pleuro-pulmonares, tais como atelectasias, pneumonias não especificas, fístula brônquica e cavidade pleural residual pós ressecção e promove, também, a reabilitação funcional destes pacientes.

\section{DISCUSSÃO}

As pesquisas relacionadas a recursos fisioterapêuticos 
em pacientes com TP parecem ser limitadas e se concentram em países como Japão, Ucrânia e Rússia.

Os resultados sugerem que o ultra-som é o recurso mais utilizado, porém, é necessário um aprofundamento maior nas pesquisas antes de ser adotado como conduta fisioterapêutica.

$\mathrm{O}$ acesso à informação quanto aos recursos fisioterapêuticos aplicados à TB pulmonar é restrito, visto que os artigos, na íntegra, estão publicados em japonês ou russo, sendo disponibilizado somente o resumo em inglês. Em muitos resumos, as descrições da pesquisa são incompletas, os resultados e tratamentos propostos não são detalhados ou até mesmo descritos.

É preocupante a dificuldade em encontrar pesquisas científicas que abordem o tratamento fisioterapêutico na TP, uma vez que ocorrem de 8 a 9 milhões de casos novos por ano no mundo ${ }^{1}$. Parte representativa desses pacientes é hospitalizada e, nos países em desenvolvimento, as internações são indicadas principalmente devido ao mau estado geral e a caquexia, o que significa uma fase adiantada da doença ${ }^{15}$.

\section{CONCLUSÃO}

A necessidade de aprofundar os conhecimentos nessa matéria é urgente, considerando a importância da Tuberculose Pulmonar e o papel significativo da fisioterapia na melhora da qualidade de vida destes pacientes.

\section{REFERÊNCIAS BIBLIOGRÁFICAS}

1. Hino P. Distribuição espacial dos casos de tuberculose no município de Ribeirão Preto, nos anos de 1998 a 2002. [Dissertação de Mestrado]. Ribeirão Preto: Escola de Enfermagem de Ribeirão Preto da USP;2004.

2. Gutierrez RS, Santos BR, Espina CA, Azambuja HCP, Silva LCC. Tuberculose. In: Silva LCC. Condutas em pneumologia. Vol.1. Rio de Janeiro: Revinter, 2001.

3. Siqueira HR, Rafful M. Tuberculose pulmonar. In: Silveira IC. O pulmão na prática médica. $4^{\mathrm{a}}$ ed. Rio de Janeiro: Biomédicas, 2000. 4. Freitas CHO. Tuberculose. In: Oliveira JBB, Freitas CHO. Fisioterapia pneumofuncional- perguntas e respostas. Fortaleza:
Universidade de Fortaleza, 2000.

5. Veronesi R, Fcaccia R. Tratado de infectologia. Rio de Janeiro: Atheneu, 2002.

6. Lomachenkov VD, Kuprikova IM, Golubeva VS, Pochtennyi IT, Nikolaeva GM. Effectiveness of complex treatment in combination with short-wave therapy and protease inhibitors in first detected patients with pulmonary tuberculosis. Probl Tuberk 1997; 1: 42-4.

7. Samosiuk IZ, Miasnikov VG, Klimenko IV. The use of lowfrequency ultrasound in the combined therapy of pulmonary tuberculosis patients. Vopr Kurortol Fizioter Lech Fiz Kult 1999 Mar-Apr; 2: 9-11.

8. Balasaniants GS. Effectiveness of the use of ultrasound irradiation of the spleen in patients with acutely progressive pulmonary tuberculosis. Probl Tuberk 2002; 6: 24-30.

9. Savchenko VI, Samosiuk IZ. Effect of magnetic and ultrasound therapy on clinical manifestations and general parameters of efficiency in the treatment of pulmonary tuberculosis. Lik Sprava 2004 Oct-Nov; 7: 41-6.

10. Klymenko IV. An analysis of the manifestations of the side effects of low-frequency ultrasonic therapy in pulmonary tuberculosis patients. Lik Sprava 1999 Oct-Dec; 7-8: 99-102. 11. Tada A, Matsumoto H, Soda R, Endo S, Kawai H, Kimura G, et al. Effects of pulmonary rehabilitation in patients with pulmonary tuberculosis sequelae. Nihon Kokyuki Gakkai Zasshi 2002 Apr; 40 (4): 275-81.

12. Levchenko GI. Physiotherapy in the treatment of pulmonary tuberculosis. Voen Med Zh 2002 May; 323(5): 49-51, 112.

13. Puri MM, Arora VK. Role of gallium arsenide laser irradiation at $890 \mathrm{~nm}$ as an adjunctive to anti-tuberculosis drugs in the treatment of pulmonary tuberculosis. Indian J Chest Dis Allied Sci 2003 Jan-Mar; 45 (1): 19-23.

14. Strelis AA, Strelis AK, Roskoshnykh VK. Vibration massage in the prevention of postresection complications and in the clinical rehabilitation of patients with pulmonary tuberculosis after surgical interventions. Probl Tuberk Bolezn Legk 2004; 11: 29-34.

15. Nogueira, PA. Motivos e tempo de internação e o tipo de saída em hospitais de tuberculose no Estado de São Paulo, Brasil1981 a 1995. J Pneumol 2001 Mai-Jun; 27(3): 123-29.

Endereço para correspondência: Maria Nazareth Vianna Roseiro Rua Paulo Tinoco Cabral 488 Ribeirão Preto-SP CEP: 14020-270

Fone/Fax: 016-3623-3200

nroseiro@uol.com.br 